Review

\title{
Q2 The innate immune system, toll-like receptors and dermal wound healing: A review
}

\author{
M.J. Portou ${ }^{\text {a, } *}$, D. Baker ${ }^{\text {a }}$, D. Abraham ${ }^{\text {b }}$, J. Tsui ${ }^{\text {a }}$ \\ a Royal Free Vascular, Division of Surgery and Interventional Science, Royal Free Campus, UCL, London, UK \\ b Centre for Rheumatology and Connective Tissue Disease, UCL, London, UK
}

\section{Article history:}

Received 16 November 2014

Received in revised form 5 February 2015

Accepted 8 February 2015

Available online $\mathrm{xxxx}$

\section{Keywords:}

Wound healing

Innate immune system

Diabetes

\begin{abstract}
A B S T R A C T
Wound healing is a complex physiological process comprised of discrete but inter-related and overlapping 17 stages, requiring exact timing and regulation to successfully progress, yet occurs spontaneously in response to 18 injury. It is characterised by four phases, coagulation, inflammation, proliferation and remodelling. Each phase 19 is predominated by particular cell types, cytokines and chemokines. The innate immune system represents the 20 first line of defence against invading microorganisms. It is entirely encoded with the genome, and comprised 21 of a cellular response with specificity provided by pattern recognition receptors (PRRs) such as toll-like receptors 22 (TLRs). TLRs are activated by exogenous microbial pathogen associated molecular patterns (PAMPs), initiating an 23 immune response through the production of pro-inflammatory cytokines and further specialist immune cell 24 recruitment. TLRs are also activated by endogenous molecular patterns termed damage associated molecular 25 patterns (DAMPs). These ligands, usually shielded from the immune system, act as alarm signals alerting the 26 immune system to damage and facilitate the normal wound healing process. TLRs are expressed by cells essential 27 to wound healing such as keratinocytes and fibroblasts, however the specific role of TLRs in this process remains 28 controversial. This article reviews the current knowledge on the potential role of TLRs in dermal wound healing 29 where inflammation arising from pathogenic activation of these receptors appears to play a role in chronic 30 ulceration associated with diabetes, scar hypertrophy and skin fibrosis.
\end{abstract}

(c) 2015 Published by Elsevier Inc. 32

\section{Contents}

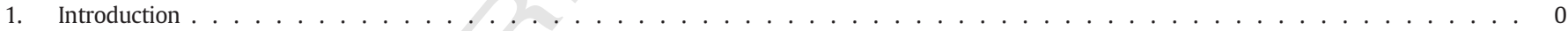

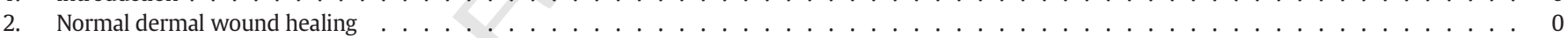

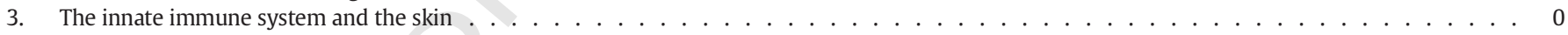

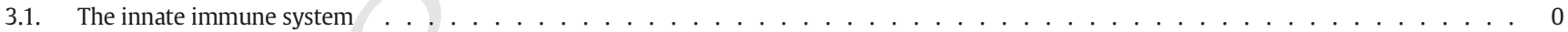

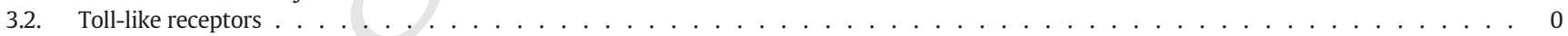

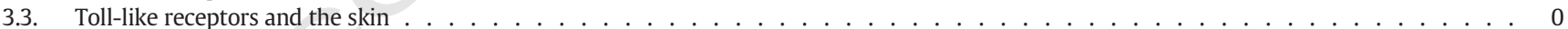

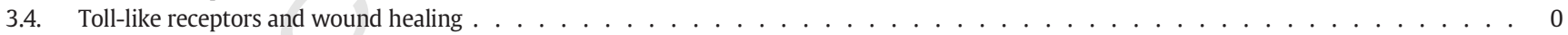

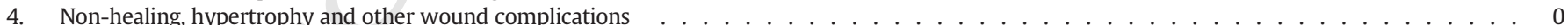

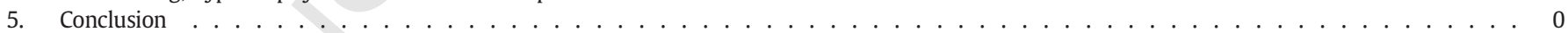

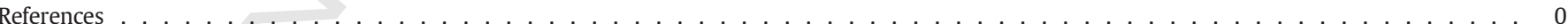

\section{Introduction}

Wound healing is a complex physiological process comprised of discrete but inter-related and overlapping stages, requiring exact timing

'This article is based on a presentation at the 8th International Workshop on Cardiovascular Biology \& Translational Medicine held in London in September 2013.'

* Corresponding author at: Royal Free Vascular, Division of Surgery and Interventional science, 9th Floor Royal Free Hospital, Pond Street, London NW3 2QG, UK. Tel.: +44 7946619670.

E-mail address: mjportou@doctors.org.uk (M.J. Portou). and regulation to successfully progress, yet occurs spontaneously in 53 response to injury. It is characterised by four phases, coagulation, in- 54 flammation, proliferation and remodelling. Each phase is predominated 55 by particular cell types, cytokines and chemokines.

Mammals and higher organisms have evolved complex immune 57 defences against pathogenic microbial organisms in the form of the 58 antibody based adaptive immune system and the innate immune sys- 59 tem, a primitive evolutionary cellular based system. Pattern recognition 60 receptors (PRRs) on the cell surface of innate immune cells recognise 61 discrete microbial molecular patterns triggering their activation termed 62 
pathogen associated molecular patterns (PAMPS). A group of highly conserved and prime PRRs are toll-like receptors (TLRs). In addition to PAMPs, TLRs also recognise a rage of endogenous self derived molecular patterns released in response to tissue and cellular damage, termed damage associated molecular patterns (DAMPs).

TLRs are expressed by cells comprising the dermis and epidermis of the skin, in addition to the immune cells that reside within the skin or those that are recruited from circulation. The activation and timing of specific TLRs and the presence of conditions affecting TLR expression and activation determine whether TLR activation promotes or inhibits the wound healing process, leading to chronic wounds.

\section{Normal dermal wound healing}

Immediately following trauma to the skin, platelets aggregate at the site of injury with haemostasis achieved following local vasoconstriction and activation of the clotting cascade, resulting in fibrin clot formation [1]. The inflammatory phase of wound healing begins with release of proinflammatory cytokines such as platelet derived growth factor (PDGF), transforming growth factor (TGF- $\beta$ ), fibroblast growth factor (FGF), epidermal growth factor (EGF) and Interleukin 8 (IL-8/CXCL-8) from the newly formed clot and directly from the damaged tissues [2]. These act as potent chemotactic signals to immediately recruit neutrophils to the wound [3]. Circulating polymorphonuclear neutrophils (PMN) begin migration within minutes from the blood into the immature wound bed formed by the clot, peaking within the first $24 \mathrm{~h}$ [4]. The neutrophils now present in the wound provide a crucial defence against microbial invasion following disruption to the skins natural barrier function, clearing both pathogen and tissue debris by phagocytosis $[2,5]$.

The process of platelet de-granulation, activation of the complement cascade, and the migration and signalling of PMNs results in the further production of chemotactic factors such as complement component 5 (C5), fibrin by products and TGF- $\beta c$ [6]. These chemokines along with chemokine ( $\mathrm{C}-\mathrm{C}$ motif) ligand 5 (CCL5) produced by keratinocytes, recruit monocytes to the wound, which under the influence of local cytokines undergo differentiation to become mature wound macrophages [6,7]. By days three to five following injury, tissue macrophages become the dominant cell type [8]. Wound macrophages continue the process of wound bed clearance through phagocytosis of apoptotic cells including the early phase PMNs, tissue debris and microbial organisms [8]. In addition, macrophages also directly aid the debridement of injured and devitalised tissue through release of protease and metalloprotease enzymes [8,9]. Over and above their phagocytic role, an important initial function of wound macrophages is the release of cytokines which further aid the recruitment and activation of inflammatory cells [2]. As the inflammatory phase progresses, macrophages produce important growth factors such as KGF, TGF- $\beta$, VEGF and PDGF which stimulate fibroblast and keratinocyte growth and migration and the process of angiogenesis [1]. It is therefore considered that macrophages are responsible for the transition to the proliferative phase of wound healing [2].

The late inflammatory phase becomes characterised by infiltration of T-lymphocytes under the influence of IL-1, which peak at day 7 after injury. At this stage there is considerable temporal overlap between the late inflammatory, proliferative and early remodelling phases of normal wound healing. [10]. As described, the inflammatory phase involves a well characterised sequence of immune cell infiltration, neutrophils followed by macrophages then finally T-lymphocytes [2].

Like macrophages, T-lymphocytes appear to have a complex yet significant role in the normal process of wound healing, however these processes, functions and pathways remain poorly understood. Studies utilising In vivo murine knock-out models have suggested that absent or delayed T-lymphocyte wound infiltration results in an impairment of the healing process [2]. However there appears to be differential roles of CD4 + T helper and CD8 + cytotoxic T cells, with CD4 + cells found to have a positive promoting effect on healing, and CD8 + cells an inhibitory effect [11]. In addition, T-lymphocytes have a regulatory 127 effect on inflammation and fibrosis and a dermal subgroup of gamma 128 delta T cells produce keratinocyte growth factor (KGF) and insulin-like 129 growth factor 1 which stimulate keratinocyte proliferation, promoting 130 healing [12].

Central to the proliferative phase of wound healing is the formation 132 of granulation tissue. Dermal fibroblast proliferation, migration and 133 differentiation (into contractile myofibroblasts) occurs under the influ- 134 ence of growth factors such as fibronectin, PDGF, FGF, TGF- $\beta$ and C5a, as 135 inflammatory cytokine release diminishes [13]. Fibroblasts are crucial 136 for the production of extracellular matrix comprised of collagen, glycos- 137 aminoglycans, proteoglycans, fibronectin and elastin [14]. Angiogenesis 138 occurs as dermal endothelial cells migrate into the newly forming extra- 139 cellular matrix under the influence of macrophage derived angiogenic 140 factor, forming new capillaries [8].

During the proliferative phase, wound contraction is an important 142 process that occurs through the action of myofibroblasts, differentiated 143 from mesenchymal fibroblast cell lines [15]. Myofibroblasts, unlike 144 fibroblasts express the contractile protein $\alpha$ smooth muscle actin 145 $(\alpha \mathrm{SMA})$ and as the wound matures are gradually lost from the granula- 146 tion tissue [15].

147

Restoration of the skins crucial barrier function requires successful 148 epidermal keratinocyte migration, proliferation and differentiation to 149 cover the newly formed granulation tissue and extracellular matrix in 150 a process termed re-epithelialisation [16]. In intact skin, keratinocytes 151 are closely attached to adjacent epithelial cells through desmosomes, 152 and to the extra cellular matrix of the underlying basement membrane 153 by hemidesmosomes [17]. Following injury, keratinocytes become mo- 154 bilized by undergoing phenotypic changes favouring detachment in a 155 process that remains incompletely understood. However cytokines 156 such as IL-1, IL-6 and TNF- $\alpha$ produced in inflammatory phase seem to 157 help modulate the migratory phenotype of keratinocytes [17]. Migration 158 and proliferation are influenced by growth factors such as IGF1 and epi- 159 dermal growth factors (EGF) [18]. In addition, EGF, KGF and TGF- $\beta$ have 160 important pro-migratory or pro-proliferative effects on keratinocytes 161 [17]. Essential to the process of keratinocyte migration is production 162 of proteases such as collagenases and matrix metalloproteases [19]. 163 These degrade adhesions between the keratinocyte and the newly 164 formed extracellular matrix to permit cell movement [16]. Disruption 165 of the basement membrane after injury requires migrating keratino- 166 cytes to utilize fibronectin, vitronectin and fibrin components of the pro- 167 visional extracellular matrix for attachment through focal integrin 168 receptors [16]. Closely following migration is rapid proliferation and 169 basement membrane repair through laminin production [20]. Keratino- 170 cyte differentiation and keratin production occurs as the epidermal 171 barrier and normal stratified architecture is restored [21].

The remodelling phase is the longest phase of the wound healing 173 process, continuing for weeks to months [8]. This phase is characterised 174 by reduced proliferation and inflammation, active re-organisation of the 175 extracellular matrix and regression of the newly formed capillaries as 176 the nutrient requirements of the wound site reduce [8]. 177

Type III collagen produced by fibroblasts during the proliferative 178 phase is gradually replaced by structural type I collagen, through the ac- 179 tion of collagenases and matrix-metalloproteases [16]. During remodel- 180 ling, collagen becomes more organised and increasingly cross-linked 181 strengthening the scar; fibronectin disappears, and hyaluronic acid 182 and glycosaminoglycans are replaced by proteoglycans. The result is 183 the re-organisation of the extracellular matrix to an architecture more 184 closely resembling normal tissue [2].

Mammals and other higher vertebrate organisms have evolved com- 188 plex immune defences against invading pathogenic microorganisms, 189 
comprised of the innate and adaptive immune systems [22]. The innate immune system is in evolutionary terms primitive and unlike the clonal selection antibody-based response of the adaptive immune system, is entirely encoded within the genome [23]. Innate immunity comprises the entire immune response of invertebrate organisms, however in higher species it provides the first line of defence against infectious pathogens and aids adaptive responses through antigen presentation, with the adaptive response concerned with later stages of infection, providing a targeted and specific response and immunological memory [22].

The innate immune system is comprised of numerous different cellular components such as neutrophils, eosinophils, basophils, mast cells, monocytes, macrophages, dendritic cells, NK cells, gamma delta T cells, B-1 cells [24]. Rather than coordinating a non-specific proinflammatory or phagocyte response, cell activation, pathogen recognition and a specificity of the innate immunity is conferred by the presence of specific receptors expressed by these immune cells termed pattern recognition receptors (PRRs) [25,26].

\subsection{Toll-like receptors}

Toll-like receptors (TLRs) are key pattern recognition receptors of the innate immune system [27]. Other examples of PRRs include scavenger receptors (SRs), C-type lectin receptors (CLRs), NOD-like receptors (NLRs) and B2 integrins [26]. These receptors are highly conserved in evolution and recognise discrete molecular components of invading pathogens termed pathogen associated molecular patterns (PAMPs), such as lipids, lipopeptides, proteins and nucleic acids [22,27]. The recognition of microbial PAMPs by PRRs leads to activation of specific signalling pathways and a variety of cell dependent responses, including pro-inflammatory cytokine release, phagocytosis and antigen presentation [26].

The Toll-like receptor family consists of thirteen identified members of which ten are expressed in humans [24]. TLRs are located either at the cell surface (TLRs 1,2, 4, 5, 6) or in the intracellular compartment (TLRs $3,7,8,9)$ primarily on exosomes and endoplasmic reticulum $[28,29]$. TLRs are transmembrane proteins consisting of an ectodomain comprising leucine-rich repeats, a transmembrane domain and an intracellular (TIR) domain [28]. The binding of TLR ligands results in activation through the recruitment of specific adaptor molecules such as myeloid differentiation factor 88 (MyD88), MyD88 adaptor like (MAL), TIR domain-containing adapter-inducing interferon- $\beta$ (TRIF) and TRIF adaptor molecule (TRAM) to the intracellular domain [28,30]. All TLRs except TLR3 utilize one of two signalling pathways, the MyD88 dependent and MyD88 independent (TRIF) pathways, resulting in the activation of nuclear transcription factors such as NFkB, JNK and MAPK [28]. TLR3 signals solely through the TRIF pathway [27]. The result is proinflammatory cytokine and type 1 interferon gene induction [22].

TLRs efficiently recognise distinct components of pathogens that are essential to their metabolism, preventing mutations rendering them undetectable [31]. TLRs 1, 2 and 6 recognise gram positive bacteria cell wall constituents such as lipoproteins, peptidoglycans and lipoteichoic acid [31]. TLR4 is activated by the gram negative bacteria cell wall component lipopolysaccharide (LPS) [32] and TLR5 bacterial flagellin [31]. The intracellular TLRs 3,7 and 8 recognise double and single stranded viral RNA, and TLR9 non-methylated CPG dinucleotides present in bacterial DNA [31,33].

In addition to exogenous microbial PAMP ligands, TLRs are also activated by a range of endogenous ligands released as a result of tissue and cellular injury termed damage associated molecular patterns (DAMPs). These are usually hidden from recognition, however following injury they are released or revealed, triggering a TLR mediated inflammatory response [31]. It has been suggested DAMPs act as danger signals, released by injured tissues, alerting the immune system of damage [34]. The resulting sterile inflammation is a key stimulator for the recruitment of innate immune inflammatory cells and initiation of the wound healing process [31]. DAMPs identified as TLR ligands 254 include the extracellular matrix constituent hyaluronic acid, HMGB1 255 (a nuclear protein), Heat shock proteins (HSPs) 60 and 70, oxidised 256 LDL, fibrinogen and fibronectin [35].

\subsection{Toll-like receptors and the skin}

Intact skin provides an external barrier to the environment, 259 preventing infection by the majority of pathogenic bacteria, viruses 260 and fungi [36]. In addition to this physical defence, cells of the innate 261 immune system present in skin such as dermal mast cells, phagocytes 262 and dendritic cells such as Langerhans cells of the epidermis, and 263 those readily recruited from blood such as neutrophils, macrophages, 264 basophils, eosinophils, NK cells and gamma-delta T cells all express 265 TLRs for pattern recognition [31]. On detection of invading microbial 266 pathogens through recognition of PAMPs, TLR activation results in the 267 initiation of a pro-inflammatory defence response, promoting phagocy- 268 tosis, immune cell recruitment and antigen presentation [36]. In addi- 269 tion to immune cells, TLRs are also widely expressed by a variety of 270 non-immune cells contained within both the epidermis and dermis 271 which are vital to wound healing [37].

The epidermis is primarily comprised of keratinocytes, which have 273 been demonstrated to express TLRs 1-6 and TLR9 and 10 [38]. Unlike 274 specialist immune cells, keratinocytes and other epithelial cells com- 275 prise the boundary and interface with the external environment and 276 are under constant exposure to microbes and PAMPS [31]. They are 277 able to maintain a delicate balance between tolerance of commensal 278 organisms and the detection of infection and injury and subsequent in- 279 flammatory response [31]. The relative expression of TLRs by keratino- 280 cytes also seems to vary depending on position of the cell, for instance 281 TLR5 is predominantly expressed in the basal layers, whereas TLR9 is 282 expressed to a greater degree by more differentiated cells of the upper 283 epidermal layers [39]. It does appear however all TLRs are functional, 284 and produce distinct immune responses [40]. For instance, activation 285 of keratinocyte TLRs 2, 3, 4, 5 and 9 by their respective ligands resulted 286 in TNF- $\alpha$, IL-8, CCL2 (basophil chemokine) and CCL20 (macrophage in- 287 flammatory protein-3) release [40]. TLR3 and TLR9 activation produced 288 CXCL9 and CXCL10, involved in T-memory cell activation and type 1289 interferon production [40].

Fibroblasts located in the dermis produce extra cellular matrix 291 constituents, cytokines, growth factors and have a crucial role in the 292 wound healing process as described above. They have been found to 293 express the full range of human TLRs from 1 to 10 [41]. Studies have 294 demonstrated in vitro activation of TLRs 2, 3, 4, 5 and 9 resulted in pro- 295 duction of interferon- $\gamma$, CXCL9, CXCL10 and CXCL11, important in the 296 recruitment of T-cells and NK cells [40]. TLR4 activation in dermal fibro- 297 blasts has been demonstrated to result in IL-6, IL-8 and monocyte 298 chemotactic protein (MCP) [42]. Microvascular cells such as dermal 299 endothelial cells have been shown to highly express TLR4 and to a lesser 300 extent TLR2. In vitro treatment with the exogenous TLR4 ligand LPS re- 301 sulted in NFkB activation. Likewise exposure to the endogenous derived 302 ligand hyaluronan induced IL-8, a potent chemokine, stimulating the 303 recognition of tissue injury and promoting initiation of the early stages 304 of the wound healing process [43].

\subsection{Toll-like receptors and wound healing}

As previously described, recognition of endogenous ligands by TLRs 307 on both immune and non-immune cells of the skin provide alarm 308 signals via TLR activation and resulting sterile inflammation alerting to 309 tissue injury. However, the effect of TLR activation on the wound 310 healing process extends beyond the initial recognition of cellular 311 damage, and it appears depending on the location, timing and degree 312 of activation may have a promoting or inhibiting effect on the process 313 of wound healing and tissue regeneration [44] (Table 1). 
t1.1 Table 1

t1.2 Summary of TLR wound healing studies.

\begin{tabular}{|c|c|c|c|c|}
\hline Study & TLR & Model & Wound & Findings \\
\hline Dasu et al. (2010) [44] & 2 & In vivo murine, knock out & Diabetic & TLR2 knock out was beneficial for wound healing in diabetes induced animals \\
\hline Dasu et al. (2013) [45] & 4 & In vivo murine, knock out & Diabetic & TLR4 knock out improves wound healing and reduces inflammation in diabetic mice \\
\hline Suga et al. (2013) [7] & 2 and 4 & In vivo murine, knock out & Non-diabetic & $\begin{array}{l}\text { TLR } 2 \text { and } 4 \text { knock out impaired wound healing at days } 3 \text { and 7. TLR4 rather than TLR2 } \\
\text { regulates healing through TGF- } \beta \text { and CCL5 }\end{array}$ \\
\hline Chen et al. (2013) [43] & 4 & In vitro, In vivo murine, knock out & Non-diabetic & $\begin{array}{l}\text { Injury stimulates TLR4 mRNA expression in keratinocytes. } \\
\text { Wound healing is prolonged in TLR4 deficient mice. }\end{array}$ \\
\hline Sato et al. (2010) [31] & 9 & In vivo murine, knock out & Non-diabetic & $\begin{array}{l}\text { Wounds treated with TLR9 agonists exhibit accelerated healing. } \\
\text { TLR9 deficient animals demonstrate delayed wound healing }\end{array}$ \\
\hline Lin et al. (2011) [47] & 3 & In vivo murine & Non-diabetic & Wound healing is significantly delayed in TLR3 deficient mice compared to wild type \\
\hline Lin et al. (2012) [48] & 3 & In vivo murine, human & Non-diabetic & $\begin{array}{l}\text { Topical application of TLR3 agonist accelerated wound healing when applied to human } \\
\text { and mouse wounds } \\
\text { TLR3 deficiency inhibited wound healing }\end{array}$ \\
\hline
\end{tabular}

In vitro and in vivo data has suggested that TLR4 becomes upregulated within the first $12-24 \mathrm{~h}$ following injury and slowly decreases to baseline at day 10 , and is primarily concentrated in epidermal keratinocytes [45]. The same study demonstrated significantly impaired wound healing in TLR4 deficient mice at days $1-5$, with no difference seen from wild type at 10 days [45]. An altered pattern of cytokine release and inflammatory cell infiltration was observed with decreased IL-1 $\beta$ and IL-6, and an increase in neutrophil, macrophage and T-cell infiltrates in the wounds of knockout animals at discreet time points [45]. Another study also observed impairment in wound healing in TLR2 and TLR4 deficient mice at days 3 and 7, but observed a decrease in neutrophil and macrophage infiltration, and reduced TGF- $\beta$ and CCL5 expression [7]. Activation of TLR4 and TLR2 appears therefore to have a beneficial effect on wound healing in the early stages following acute injury, at least in absence of other influences on TLR expression, signalling and activation.

However the story does not end there. Controversy exists as to the exact effect of TLR4 and TLR2 in the wound healing process. Given the seemingly important regulatory role of TLR4 and TLR2 in initiating the early stages of wound healing, it perhaps seems counter-intuitive that wound healing was significantly improved in TLR2 deficient mice with induced diabetes compared to diabetic wild-type animals [46]. The same effect was also observed in diabetic TLR4 deficient mice [47] in apparent contradiction of the studies described above.

In addition to decreased healing time, the wounds from TLR2 deficient mice also demonstrated significantly reduced NFkB activation, IL-6 and TNF- $\alpha$ release [46]. In the same study when comparing wildtype diabetic mice to non-diabetic controls, TLR2 mRNA and protein expression was significantly increased, along with markers of activation such as increased expression of MyD88, IRAK and NFkB [46]. Likewise, TLR4 mRNA and protein expression, IL-6, TNF- $\alpha$ and NFkB activation was increased in wild-type diabetic compared to non-diabetic animals, with a corresponding reduction in IL-6, TNF- $\alpha$ and NFkB activity in the TLR4 deficient diabetic populations [47].

These studies demonstrated significantly increased TLR2, TLR4 and MyD88 expression in diabetic compared to non-diabetic wounds and suggests in diabetes, TLR2 and TLR4 mediated hyperinflammation results in an impairment of wound healing. Persistent activation of TLR2 and TLR4 is also associated with other chronic non-healing wounds such as chronic venous ulceration [48].

Wound healing studies utilising TLR3 deficient mice resulted in significantly delayed wound healing compared to wild-type controls, led to decreased neutrophil and macrophage recruitment, and reduced CXCL2, CCL2 and CCL3 chemokines [49]. Further to this effect, the TLR3 agonist poly(I:C) significantly accelerated wound healing when applied topically to human and mouse wounds compared to control and resulted in greater neutrophil and macrophage recruitment and upregulated CXCL2 [50].

TLR9 deficient mice demonstrated delayed wound healing compared to wild-type [33]. In addition, topical administration of the TLR9 agonist CpG ODN to wounds resulted in significantly improved healing 365 times, increased macrophage infiltration and increased production of 366 VEGF [33].

\section{Non-healing, hypertrophy and other wound complications}

Chronic wounds such as foot ulceration are a frequent and challeng- 369 ing complication of diabetes with a life time risk of between 15 and $25 \% 370$ [51,52]. This translates to a $20 \times$ greater risk of major amputation 371 compared to non-diabetics, and remains the most common cause of 372 hospitalisation amongst diabetic patients [6]. Diabetic foot ulcers are 373 multifactorial in causation, although a predomination for either neurop- 374 athy or ischaemia often exists [6]. The result is a wound characterised by 375 poor healing, with the progression of the normal process stalled, or 376 failed to initiate, leading to a chronic, static wound.

The diabetic wound environment differs from the normal acute 378 wound process through a prolonged and persistent inflammatory 379 phase (Fig. 1). There is an exaggerated and sustained neutrophil and 380 macrophage infiltration, which in a db/db mouse model was demon- 381 strated to be associated with deregulated and prolonged chemokine 382 expression, such as macrophage inflammatory protein 2 and macro- 383 phage chemoattractant protein 1 [53].

Although the initial infiltration of immune cells is impaired, once 385 activated the result is a hyperinflammatory response with elevated in- 386 flammatory cytokine production of TNF- $\alpha$, IL-1 $\beta$ and IL-6, and increased 387 NFkB regulated matrix metalloprotease (MMP) production leading to 388 excessive extracellular matrix destruction and grossly impaired granu- 389 lation tissue formation [6]. Neutrophils in particular appear to con- 390 tribute to these destructive wound conditions through upregulated 391 release of MMP-8 and downregulated release of the MMP inhibitor 392 TIMP1 in chronic wounds [54]. The resulting hostile environment of 393 excess inflammatory cytokine production (TNF- $\alpha$, IL-6) also impairs 394 other events and processes crucial to healing such as fibroblast migra- 395 tion and proliferation, collagen synthesis and promotes apoptosis in 396 fibroblasts and vascular precursor cells [6]. 397

Diabetic ulceration is an example of chronic inflammation directly 398 leading to a significant impairment in the healing process and the crea- 399 tion of a chronic non-healing wound. As previously described, there is 400 compelling evidence this pathological inflammation is mediated via ex- 401 cessive TLR activation. Another consequence of abnormal TLR mediated 402 inflammation on the wound healing process is in over healing in the 403 form of hypertrophic scar formation [42]. Hypertrophic scars develop 404 following trauma as a result of excessive production of ECM compo- 405 nents such as collagen, and although the mechanism remains unclear, 406 are associated with prolonged inflammation and bacterial contamina- 407 tion [42]. Comparison of hypertrophic and normal scar tissue from 408 burns patients demonstrated increased TLR4 staining in hypertrophic 409 tissues and increased TLR4 and MyD88 mRNA in fibroblasts isolated 410 from hypertrophic scars [42]. A corresponding increase in pro- 411 inflammatory cytokines such as PGE2, IL-6, IL-8 and MCP-1 were also 412 


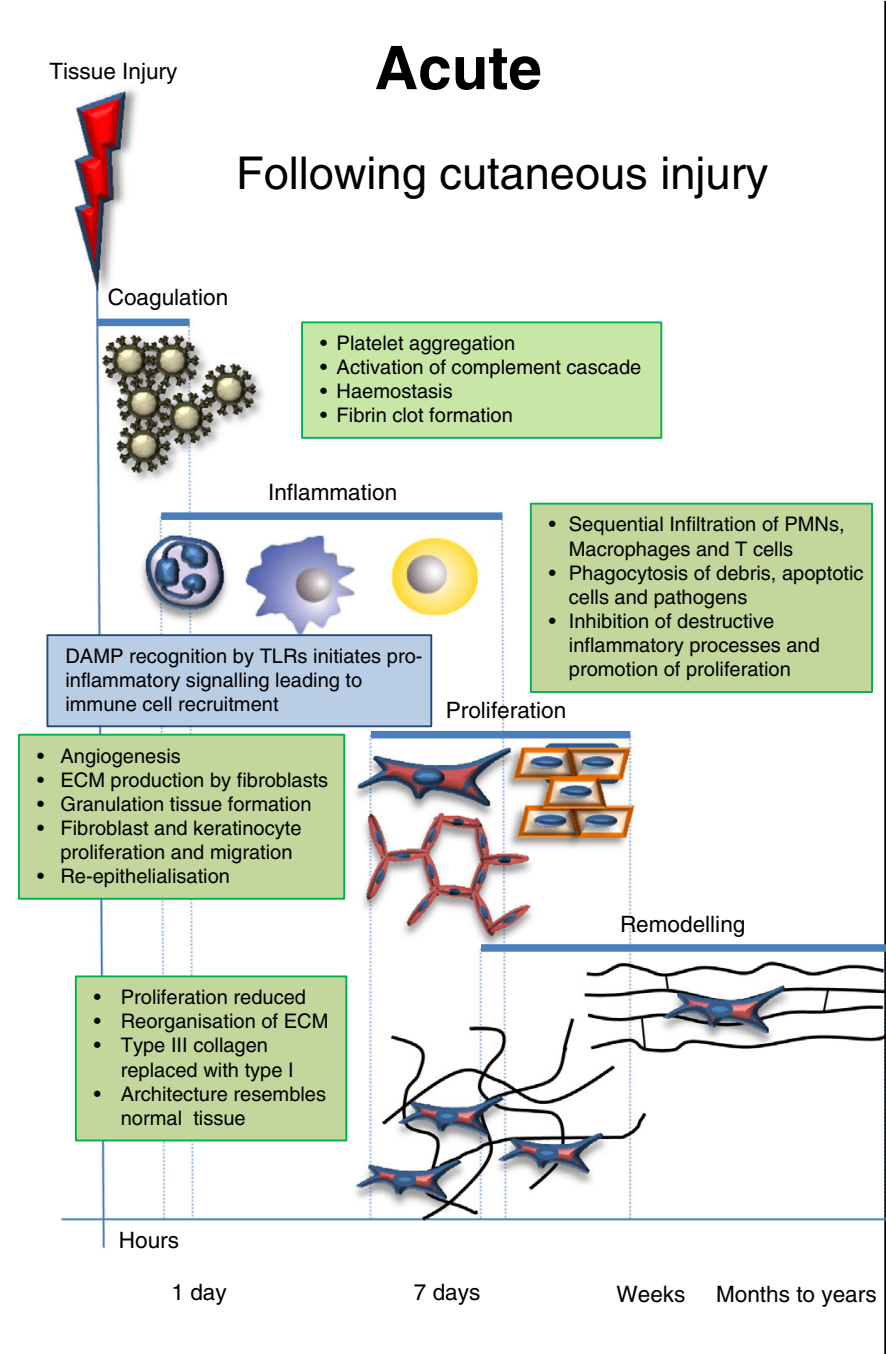

\section{Chronic}

\section{Non healing Diabetic foot ulcers}
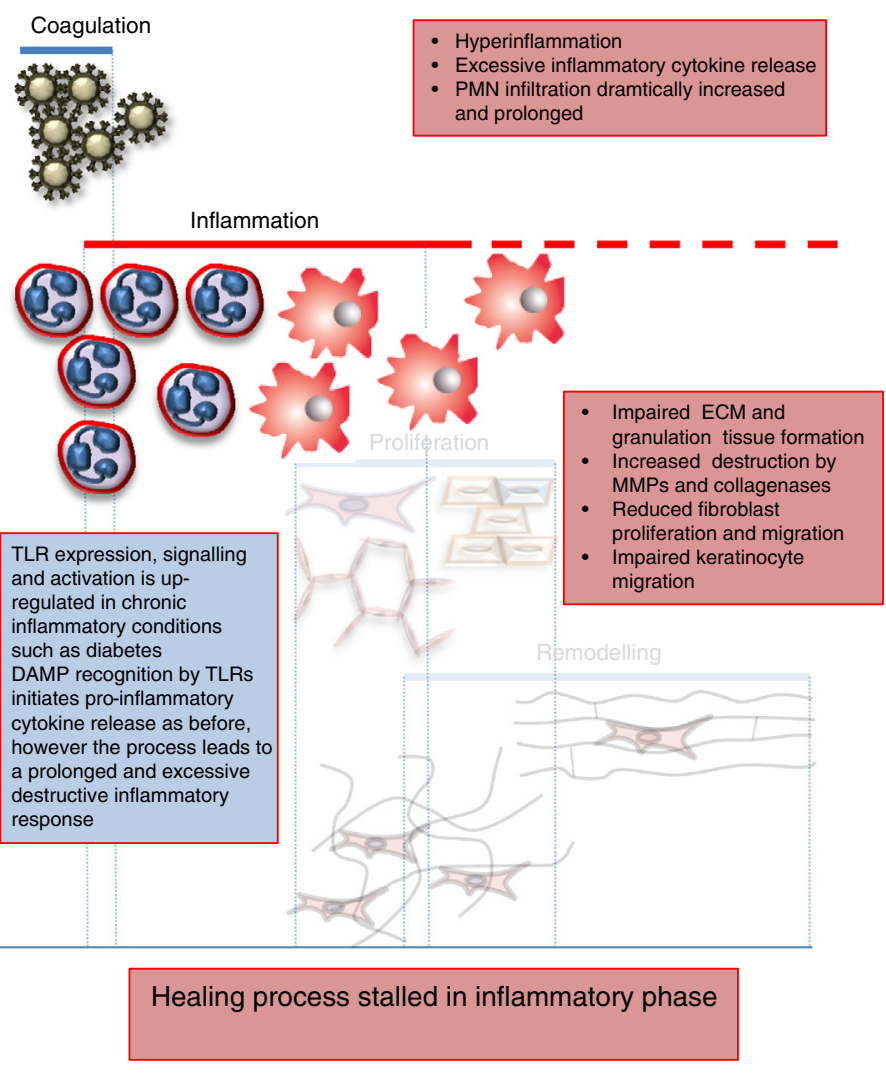

detected. It is therefore suggested that persistent TLR4 activation in dermal fibroblasts leads to hypertrophic scarring, possibly through the increased production of growth factors by supporting cells [42].

Another example of a maladaptive healing response that occurs in response to chronic inflammation is fibrosis. TLR activation is implicated in fibrotic abnormal healing responses in specific organs such as the liver, following repeated tissue injury [31]. TLR4 in particular is thought to contribute to the fibrotic reaction through chronic activation from the continuous translocation of gut bacteria associated with chronic liver diseases, and as demonstrated by the protective effect of TLR4 knock out in animal models of liver injury [31]. There does however appear to be a differential TLR effect on fibrosis depending on the organ involved, with TLR9 implicated in lung fibrosis, and TLRs 2 and 4 likely to have a greater effect in acute renal inflammation rather than chronic kidney fibrotic responses, where evidence is conflicting [31].

The role of TLRs in fibrotic skin reactions has been of particular interest in conditions such as systemic sclerosis. TLR4 activation has been implicated by murine models of skin fibrosis utilising bleomycin, through increased hyaluronan production, a potent TLR4 endogenous ligand [55]. In addition, studies utilising human tissue biopsies from scleroderma patients have demonstrated TLR4 and associated adaptor molecules are overexpressed in affected skin, and correlate with disease progression [56]. In vitro studies in ex-planted scleroderma fibroblasts have shown activation of TLR4 resulted in increased collagen production and gene expression of factors associated with ECM production
Fig. 1.

and remodelling, in addition to an increased susceptibility to the effects 438 of TGF- $\beta$ [57]. Recent work has also identified TLR4 as a crucial mecha- 439 nism through which in scleroderma, injured keratinocytes interact with 440 fibroblasts through increased production of the protein S100A9, a 441 known ligand of TLR4, leading to increased production of the pro- 442 fibrotic gene CCN2 [58].

It is therefore proposed that in chronic fibrotic skin diseases such as 444 scleroderma, persistent TLR4 activation through endogenous ligand 445 stimulation results in altered response to TGF- $\beta$, and subsequent 446 dysregulated production and remodelling of the extracellular matrix, 447 leading to profound skin fibrosis.

\section{Conclusion}

Wounds that fail to heal, such as chronic diabetic ulcers, do not prog- 450 ress through the normal stages of the healing process described in detail 451 earlier in this review. It is clear the innate immune system and the 452 pattern recognition receptors that confer specificity such as toll-like re- 453 ceptors have a crucial role in the initiation and regulation of normal 454 wound healing, however the role of the innate immune response in 455 chronic wounds remains controversial.

In addition to the excess morbidity and mortality associated with 457 foot ulceration and subsequent amputation, and with the global burden 458 of diabetes set to reach 350 million people, non-healing wounds 459 of all aetiology are set to remain an enormous economic liability for 460 
healthcare systems around the world. Manipulation of the innate immune response therefore represents a potential novel therapeutic opportunity to reduce the hyperinflammation associated with chronic wounds, and to restart the normal wound healing process.

The impact of dysregulated TLR activation and subsequent chronic inflammation on the wound healing process appears to be significantly more complex however, when the pathological yet intuitively opposed outcomes of non-healing, hypertrophy and fibrosis can all occur in different disease phenotypes within the same tissue, the skin.

\section{References}

[1] Hubner G, Brauchle M, Smola H, Madlener M, Fassler R, Werner S. Differential regulation of pro-inflammatory cytokines during wound healing in normal and glucocorticoid-treated mice. Cytokine 1996;8(7):548-56.

[2] Guo S, Dipietro LA. Factors affecting wound healing. J Dent Res 2010;89(3):219-29.

3] Roupe KM, Nybo M, Sjobring U, Alberius P, Schmidtchen A, Sorensen OE. Injury is a major inducer of epidermal innate immune responses during wound healing. J Invest Dermatol 2010;130(4):1167-77.

[4] Kim MH, Liu W, Borjesson DL, Curry FR, Miller LS, Cheung AL, Liu FT, Isseroff RR, Simon SI. Dynamics of neutrophil infiltration during cutaneous wound healing and infection using fluorescence imaging. J Invest Dermatol 2008;128(7):1812-20.

[5] Segal AW. How neutrophils kill microbes. Annu Rev Immunol 2005;23:197-223.

[6] Acosta JB, del Barco DG, Vera DC, Savigne W, Lopez-Saura P, Guillen Nieto G, Schultz GS. The pro-inflammatory environment in recalcitrant diabetic foot wounds. Int Wound J 2008;5(4):530-9.

[7] Suga H, Sugaya M, Fujita H, Asano Y, Tada Y, Kadono T, Sato S. TLR4, rather than TLR2, regulates wound healing through TGF-beta and CCL5 expression. J Dermatol Sci 2014;73(2):117-24.

[8] DiPietro LA. Wound healing: the role of the macrophage and other immune cells. Shock 1995:4(4):233-40.

[9] Deodhar AK, Rana RE. Surgical physiology of wound healing: a review. J Postgrad Med 1997;43(2):52-6.

[10] Fishel RS, Barbul A, Beschorner WE, Wasserkrug HL, Efron G. Lymphocyte participation in wound healing. Morphologic assessment using monoclonal antibodies. Ann Surg 1987;206(1):25-9.

[11] Park JE, Barbul A. Understanding the role of immune regulation in wound healing. Am J Surg 2004;187(5A):11S-6S.

[12] Jameson J, Havran WL. Skin gammadelta T-cell functions in homeostasis and wound healing. Immunol Rev 2007;215:114-22.

[13] Traversa B, Sussman G. The role of growth factors, cytokines and proteases in wound management. Prim Intention 2001;9(4):161-7.

[14] Wild T, Rahbarnia A, Kellner M, Sobotka L, Eberlein T. Basics in nutrition and wound healing. Nutrition 2010;26(9):862-6.

[15] Gilbane AJ, Denton CP, Holmes AM. Scleroderma pathogenesis: a pivotal role for fibroblasts as effector cells. Arthritis Res Ther 2013;15(3):215

[16] O'Toole EA. Extracellular matrix and keratinocyte migration. Clin Exp Dermatol 2001;26(6):525-30.

[17] Raja Sivamani K, Garcia MS, Isseroff RR. Wound re-epithelialization: modulating keratinocyte migration in wound healing. Front Biosci 2007;12:2849-68.

[18] Haase I, Evans R, Pofahl R, Watt FM. Regulation of keratinocyte shape, migration and wound epithelialization by IGF-1- and EGF-dependent signalling pathways. J Cell Sci 2003;116(Pt 15):3227-38

[19] Martins VL, Caley M, O'Toole EA. Matrix metalloproteinases and epidermal wound repair. Cell Tissue Res 2013;351(2):255-68.

[20] Amano S, Akutsu N, Ogura Y, Nishiyama T. Increase of laminin 5 synthesis in human keratinocytes by acute wound fluid, inflammatory cytokines and growth factors, and lysophospholipids. Br J Dermatol 2004;151(5):961-70.

[21] Usui ML, Mansbridge JN, Carter WG, Fujita M, Olerud JE. Keratinocyte migration, proliferation, and differentiation in chronic ulcers from patients with diabetes and normal wounds. J Histochem Cytochem 2008;56(7):687-96.

[22] Akira S, Uematsu S, Takeuchi O. Pathogen recognition and innate immunity. Cell 2006;124(4):783-801.

[23] Staros EB. Innate immunity: new approaches to understanding its clinical significance. Am J Clin Pathol 2005;123(2):305-12.

[24] Spirig R, Tsui J, Shaw S. The emerging role of TLR and innate immunity in cardiovascular disease. Cardiol Res Pract 2012;2012:181394.

[25] Gordon S. Pattern recognition receptors: doubling up for the innate immune response. Cell 2002;111(7):927-30.

[26] Areschoug T, Gordon S. Pattern recognition receptors and their role in innate immunity: focus on microbial protein ligands. Contrib Microbiol 2008;15:45-60.

[27] Patel H, Shaw SG, Shi-Wen X, Abraham D, Baker DM, Tsui JC. Toll-like receptors in ischaemia and its potential role in the pathophysiology of muscle damage in critical limb ischaemia. Cardiol Res Pract 2012;2012:121237.

[28] Navi A, Patel H, Shaw S, Baker D, Tsui J. Therapeutic role of toll-like receptor modification in cardiovascular dysfunction. Vascul Pharmacol 2013;58(3):231-9.

[29] Mann DL. The emerging role of innate immunity in the heart and vascular system: for whom the cell tolls. Circ Res 2011;108(9):1133-45.
[30] De Nardo D, De Nardo CM, Nguyen T, Hamilton JA, Scholz GM. Signaling crosstalk 537 during sequential TLR4 and TLR9 activation amplifies the inflammatory response 538 of mouse macrophages. J Immunol 2009;183(12):8110-8. 539

[31] Huebener P, Schwabe RF. Regulation of wound healing and organ fibrosis by toll-like 540 receptors. Biochim Biophys Acta 2013:1832(7):1005-17. 541

[32] Alexander C, Rietschel ET. Bacterial lipopolysaccharides and innate immunity. 542 J Endotoxin Res 2001;7(3):167-202.

[33] Sato T, Yamamoto M, Shimosato T, Klinman DM. Accelerated wound healing 544 mediated by activation of toll-like receptor 9 . Wound Repair Regen 2010;18(6): 545 586-93.

[34] Matzinger P. The danger model: a renewed sense of self. Science 2002;296(5566): 547 $301-5$

[35] Dasu MR, Ramirez S, Isseroff RR. Toll-like receptors and diabetes: a therapeutic 549 perspective. Clin Sci (Lond) 2012;122(5):203-14.

[36] Terhorst D, Kalali BN, Ollert M, Ring J, Mempel M. The role of toll-like receptors in 551 host defenses and their relevance to dermatologic diseases. Am J Clin Dermatol 552 2010;11(1):1-10.

[37] Pasparakis M, Haase I, Nestle FO. Mechanisms regulating skin immunity and 554 inflammation. Nat Rev Immunol 2014;14(5):289-301. 555

[38] Miller LS, Modlin RL. Human keratinocyte toll-like receptors promote distinct 556 immune responses. J Invest Dermatol 2007;127(2):262-3. 557

[39] Miller LS, Sorensen OE, Liu PT, Jalian HR, Eshtiaghpour D, Behmanesh BE, Chung W, 558 Starner TD, Kim J, Sieling PA, et al. TGF-alpha regulates TLR expression and function 559 on epidermal keratinocytes. J Immunol 2005;174(10):6137-43. 560

[40] Miller LS, Modlin RL. Toll-like receptors in the skin. Semin Immunopathol 2007; 561 29(1):15-26. 562

[41] Jang S, Park JS, Won YH, Yun SJ, Kim SJ. The expression of toll-like receptors (TLRs) in 563 cultured human skin fibroblast is modulated by histamine. Chonnam Med J 2012; 564 48(1):7-14.

[42] Wang J, Hori K, Ding J, Huang Y, Kwan P, Ladak A, Tredget EE. Toll-like receptors 566 expressed by dermal fibroblasts contribute to hypertrophic scarring. J Cell Physiol 567 2011;226(5):1265-73.

[43] Taylor KR, Trowbridge JM, Rudisill JA, Termeer CC, Simon JC, Gallo RL. Hyaluronan 569 fragments stimulate endothelial recognition of injury through TLR4. J Biol Chem 570 2004;279(17):17079-84.

[44] Dasu MR, Isseroff RR. Toll-like receptors in wound healing: location, accessibility, 572 and timing. J Invest Dermatol 2012;132(8):1955-8. 573

[45] Chen L, Guo S, Ranzer MJ, DiPietro LA. Toll-like receptor 4 has an essential role in 574 early skin wound healing. J Invest Dermatol 2013;133(1):258-67. 575

[46] Dasu MR, Thangappan RK, Bourgette A, DiPietro LA, Isseroff R, Jialal I. TLR2 576 expression and signaling-dependent inflammation impair wound healing in diabet- 577 ic mice. Lab Invest 2010;90(11):1628-36.

477] Dasu MR, Jialal I. Amelioration in wound healing in diabetic toll-like receptor-4 579 knockout mice. J Diabetes Complications 2013;27(5):417-21. 580

[48] Pukstad BS, Ryan L, Flo TH, Stenvik J, Moseley R, Harding K, Thomas DW, Espevik T. 581 Non-healing is associated with persistent stimulation of the innate immune 582 response in chronic venous leg ulcers. J Dermatol Sci 2010;59(2):115-22. 583

[49] Lin Q, Fang D, Fang J, Ren X, Yang X, Wen F, Su SB. Impaired wound healing with de- 584 fective expression of chemokines and recruitment of myeloid cells in TLR3-deficient 585 mice. J Immunol 2011;186(6):3710-7.

[50] Lin Q, Wang L, Lin Y, Liu X, Ren X, Wen S, Du X, Lu T, Su SY, Yang X, et al. Toll-like 587 receptor 3 ligand polyinosinic:polycytidylic acid promotes wound healing in 588 human and murine skin. J Invest Dermatol 2012;132(8):2085-92. 589

[51] Fadini GP, Albiero M, Menegazzo L, Boscaro E, Pagnin E, Iori E, Cosma C, Lapolla A, 590 Pengo V, Stendardo M, et al. The redox enzyme p66Shc contributes to diabetes 591 and ischemia-induced delay in cutaneous wound healing. Diabetes 2010;59(9): 592 2306-14.

[52] O'Loughlin A, McIntosh C, Dinneen SF, O'Brien T. Review paper: basic concepts to 594 novel therapies: a review of the diabetic foot. Int J Low Extrem Wounds 2010; 595 9(2):90-102.

[53] Wetzler C, Kampfer H, Stallmeyer B, Pfeilschifter J, Frank S. Large and sustained 597 induction of chemokines during impaired wound healing in the genetically diabetic 598 mouse: prolonged persistence of neutrophils and macrophages during the late 599 phase of repair. J Invest Dermatol 2000;115(2):245-53.

[54] Nwomeh BC, Liang HX, Cohen IK, Yager DR. MMP-8 is the predominant collagenase 601 in healing wounds and nonhealing ulcers. J Surg Res 1999;81(2):189-95. 602

[55] Yoshizaki A, Iwata Y, Komura K, Ogawa F, Hara T, Muroi E, Takenaka M, Shimizu K, 603 Hasegawa M, Fujimoto M, et al. CD19 regulates skin and lung fibrosis via toll-like 604 receptor signaling in a model of bleomycin-induced scleroderma. Am J Pathol 605 2008;172(6):1650-63.

[56] Stifano G, Affandi AJ, Mathes AL, Rice LM, Nakerakanti S, Nazari B, Lee J, Christmann 607 RB, Lafyatis R. Chronic toll-like receptor 4 stimulation in skin induces inflammation, 608 macrophage activation, transforming growth factor beta signature gene expression, 609 and fibrosis. Arthritis Res Ther 2014;16(4):R136.

[57] Bhattacharyya S, Kelley K, Melichian DS, Tamaki Z, Fang F, Su Y, Feng G, Pope RM, 611 Budinger GR, Mutlu GM, et al. Toll-like receptor 4 signaling augments transforming 612 growth factor-beta responses: a novel mechanism for maintaining and amplifying 613 fibrosis in scleroderma. Am J Pathol 2013;182(1):192-205.

[58] Nikitorowicz-Buniak J, Shiwen X, Denton CP, Abraham D, Stratton R. Abnormally 615 differentiating keratinocytes in the epidermis of systemic sclerosis patients show 616 enhanced secretion of CCN2 and S100A9. J Invest Dermatol 2014. 